\title{
Evaluation of the Obesity Genes FTO and MC4R for Contribution to the Risk of Large Artery Atherosclerotic Stroke in a Chinese Population
}

\author{
Zhi Song Lingling Qiu Zhongyang Hu Jia Liu Ding Liu Deren Hou \\ Department of Neurology, Third Xiangya Hospital, Central South University, Changsha, China
}

\section{Key Words}

FTO $\cdot$ MC4R - Obesity prone gene $\cdot$ Large artery atherosclerotic (LAA) stroke - Genetic risk factor · Synergistic effect

\begin{abstract}
Background: Obesity is a well-established risk factor for large artery atherosclerotic (LAA) stroke. The aim of the study was to explore whether obesity genes, such as MC4R and FTO, contribute to LAA stroke risk in the Chinese Han population. Methods: 322 LAA stroke patients and 473 controls were recruited. Gene polymorphism of MC4R (rs17782313) and FTO (rs8050136 and rs9939609) were genotyped. Results: No differences were observed in geno-

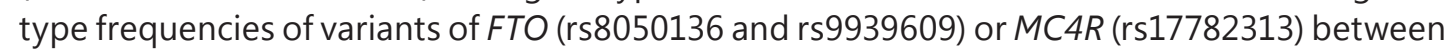
LAA stroke patients and control subjects. However, rs 17782313 of the MC4R gene was associated with LAA stroke susceptibility in smokers ( $r$ 17782313: $p=0.020, O R(95 \% \mathrm{CI})=1.55$ (1.07-2.23)) in the stratified analysis. Furthermore, multifactor dimensionality reduction analysis revealed that the combination of MC4R variant (rs17782313), hypertension and smoking habit was significantly associated with increased risk of LAA stroke ( $<<0.0001$, OR (95\% CI) $=6.57$ (4.79-9.01)). Conclusion: Our study indicated that the synergistic effects of MC4R variants, hypertension, and smoking habit contribute significantly to the risk of LAA stroke in the Chinese Han population. The finding revealed that obesity gene MC4R contribute to the risk of LAA stroke via a synergistic mechanism, which will provide new insight into the genetic architecture of LAA stroke.


Song et al.: Evaluation of the Obesity Genes FTO and MC4R for Contribution to the Risk of Large Artery Atherosclerotic Stroke in a Chinese Population

\section{Introduction}

Cerebrovascular disease is one of the three main causes of death and disability world wide. Cerebral infarction is the most common type of CVD, which is recognized as a complex disease with genetic predisposition and environmental risk factors [1,2]. There was increasing evidence to indicate that overweight/obesity is an independent risk factor for cardiovascular diseases, especially for cerebral infarction [3, 4]. Furthermore, a recent MRI-based body fat quantification study discovered the association between different patterns of fat distribution and atherosclerosis of the brain-feeding arteries, indicating the importance of visceral adiposity as a novel risk factor for large artery atherosclerotic (LAA) stroke [5]. It seems that overweight/obesity is regarded as a pivotal risk factor for LAA stroke. However, the underlying mechanism for the association between overweight/obesity and LAA stroke is not fully elucidated. The remarkable influences of obesity on metabolic syndrome and adipocytokines may take part in the pathogenesis [6-8]. Additionally, genetic risk factors may also play an important role in the disease (atherosclerosis) process. Furthermore, it is postulated that genetic predisposition is not only involved in the pathogenesis of vascular diseases but also represents an underlying link between obesity, metabolic syndrome, inflammation and atherosclerosis.

The heritability of obesity has long been appreciated, and the genetics of obesity has been the focus of intensive study for decades. Many obesity-prone genes, such as FTO, MC4R, TBX15-WARS2, GRB14, ADAMTS9, LY86, RSPO3 and ITPR2-SSPN, have been found in genomewide association studies (GWASs) on subjects of European ancestry [9-11]. Especially, FTO and MC4R have been found to be significantly associated with BMI in also GWASs including subjects of African descent, indicating that the FTO and MC4R were associated with BMI across populations of diverse ethnicities [12]. Furthermore, two recent studies revealed that polymorphisms of FTO and MC4R were associated with the risk for childhood obesity in a Chinese population $[13,14]$, providing evidence that FTO and $M C 4 R$ are obesity-prone genes in the Chinese Han population. Though obesity is a well-established risk factor for stroke, the association between obesity-prone genes and stroke is not entirely convinced in previous studies [15]. Since it had been reported in previous studies [9-14] that rs17782313 (mapped $188 \mathrm{~kb}$ downstream of MC4R-coding sequence), rs8050136, and rs9939609 (within FTO) were associated with obesity, we choose these three single-nucleotide polymorphisms (SNPs) as genetic markers to explore the association between obesity-prone genes and LAA stroke. The aim of the study was to explore whether the variants of the two well confirmed obesityprone genes (rs17782313 near MC4R, rs8050136 and rs9939609 within FTO) contribute to the risk for LAA stroke in the Chinese Han population, and to evaluate the contribution of the interplay of genetic and conventional risk factors to the LAA stroke risk in order to elucidate the underlying genetic architecture of LAA stroke.

\section{Material and Methods}

\section{Study Population}

A total of 795 unrelated individuals were recruited in this study: 322 LAA stroke patients and 473 controls. All of them were Chinese Han population from the Changsha area. The 322 patients with LAA stroke were prospectively recruited from those admitted to the Stroke Unit of the Department of Neurology, Third Xiangya Hospital of Central South University, between June 2011 and March 2014. Each diagnosis of a LAA stroke was made based on the results of a neurological examination using MRI according to the International Classification of Diseases, 9th Revision. The subtypes of ischemic stroke (IS) were classified based on the TOAST criteria [16]. Patients with IS related to LAA stroke were included. Patients with IS caused by lacunar stroke, cardioembolism, stroke caused by other determined causes, or stroke of undetermined cause were 
Song et al.: Evaluation of the Obesity Genes FTO and MC4R for Contribution to the

Risk of Large Artery Atherosclerotic Stroke in a Chinese Population

excluded. The following baseline characteristics and risk factors for cerebrovascular disease were recorded: age, gender, hypertension (treated or systolic blood pressure (SBP) $\geq 140 \mathrm{~mm} \mathrm{Hg}$ or diastolic blood pressure (DBP) $\geq 90 \mathrm{~mm} \mathrm{Hg}$ ), and diabetes (treated or fasting plasma glucose $\geq 7.0 \mathrm{mmol} / \mathrm{l}$ or 2-hour postprandial plasma glucose or random plasma glucose $\geq 11.1 \mathrm{mmol} / \mathrm{l}$ ). Quantitative traits, including the levels of BMI, blood pressure, fasting blood sugar and blood lipids (including total cholesterol (TC), triglycerides (TGs), low-density lipoprotein cholesterol (LDL-C) and high-density lipoprotein cholesterol (HDL-C)) were also recorded. Furthermore, lifestyle-based risk factors, including smoking and drinking status, were recorded. A total of 473 controls that had never experienced any stroke, were matched with the LAA stroke cases with respect to age, sex, and ethnic background. The control subjects reported no history of stroke, hematological disease, other cerebrovascular disease, liver ailment, nephrosis, autoimmune disease, or pregnancy. The protocols of this study were approved by the Ethics Committee of the Third Xiangya Hospital, Central South University, and all participants signed informed consent.

\section{SNP Genotyping}

Peripheral venous blood ( $5 \mathrm{ml}$ ) was collected from all participants, and genomic DNA was extracted from peripheral blood leukocytes using phenol-chloroform methods. The genotypes were determined using Sequenom iPLEX assays with allele detection by mass spectroscopy using Sequenom MassARRAY technology (Sequenom, San Diego, CA, USA) according to the manufacturer's protocol. The SNP genotyping was performed by laboratory technicians who had no prior knowledge of the subjects' disease status. The primers for PCR amplification and extension reactions were designed using MassARRAY Assay Design Software (Sequenom) (supplementary table 1, available at http://www.karger.com/ProdukteDB/produkte.asp?doi=448588). The single-base extension reaction products were separated and evaluated using a matrix-assisted laser desorption/ionization time-of-flight mass spectrometry (MALDI-TOF-MS) [17].

\section{Statistical Analysis}

Statistical analyses were performed using SPSS version 18.0 for Windows (SPSS Inc., Chicago, IL, USA). To identify potential confounders, univariate analyses were performed comparing demographic, clinical and lifestyle risk factors in LAA stroke patients and controls. An unpaired Student's t-test was used to compare the quantitative data and to determine the differences between the cases and the controls, whereas the chisquare test or Fisher's exact test was used to compare categorical data. The chi-square test was also applied to identify deviations from Hardy-Weinberg equilibrium and the differences of genotype frequency distribution between LAA stroke patients and control subjects. Additionally, the differences of genotype frequency distribution in LAA stroke patients and control subjects were analyzed in a stratified analysis according to the smoking status. Then, logistic regression analyses were done to verify the effect of the variant on LAA stroke risk after adjustment for the covariates (status of diabetes mellitus, hypertension, and overweight). The odds ratio (OR) and 95\% confidence intervals (95\% CIs) were calculated using the additive genetic model.

To identify higher-order epistatic or synergistic interactions in our samples, we used the multifactor dimensionality reduction method (MDR 3.0.2, www.epistasis.org), which is a nonparametric and genetic model-free machine that outperforms logistic regression for detecting and characterizing nonlinear interactions among discrete genetic and environmental attributes. The MDR method combines attribute selection, attribute construction, and classification with cross-validation and permutation testing to provide a comprehensive and powerful approach to detecting epistasis or synergistic effects. This method is regarded as a useful and efficient statistical tool for detecting synergistic interactions while avoiding the 'dimension curse', especially for detecting synergistic interactions in case-control studies using relatively small sample sizes [18]. Furthermore? MDR has been successfully applied to numerous different complex multifactorial diseases and is currently being adapted to GWASs [19].

We examined synergistic interactions using the MDR method to identify higher-order gene-conventional risk factor interactions in our samples; the MDR method was performed to identify whether interactions between genetic variants (rs8050136, rs9939609 and rs17782313) and conventional risk factors (status of diabetes mellitus, hypertension, overweight, and smoking habit) increase the susceptibility of LAA stroke. This result was confirmed by a permutation test of 1,000 implemented in the MDR software. P values (two-tailed) $<0.05$ were considered statistically significant. 
Table 1. Demographic and clinical characteristics of recruited individuals
Song et al.: Evaluation of the Obesity Genes FTO and MC4R for Contribution to the Risk of Large Artery Atherosclerotic Stroke in a Chinese Population

\begin{tabular}{llll}
\hline Characteristic & $\begin{array}{l}\text { Cases }(\mathrm{n}=322) \\
\text { mean } \pm \mathrm{SD}\end{array}$ & $\begin{array}{l}\text { Controls }(\mathrm{n}=473) \\
\text { mean } \pm \mathrm{SD}\end{array}$ & $\mathrm{p}$ value \\
\hline Age & $61.92 \pm 9.75$ & $60.73 \pm 9.21$ & $0.081^{\mathrm{a}}$ \\
TC, mmol/l & $4.75 \pm 1.27$ & $4.53 \pm 1.03$ & $0.006^{\mathrm{a}}$ \\
LDL-C, mmol/l & $2.64 \pm 0.89$ & $2.48 \pm 1.56$ & $0.101^{\mathrm{a}}$ \\
HDL-C, mmol/l & $1.31 \pm 0.35$ & $1.35 \pm 0.73$ & $0.381^{\mathrm{a}}$ \\
TG, mmol/l & $1.79 \pm 1.59$ & $1.62 \pm 1.49$ & $0.147^{\mathrm{a}}$ \\
BMI, kg/m ${ }^{2}$ & $23.18 \pm 2.96$ & $22.73 \pm 3.09$ & $0.046^{\mathrm{a}}$ \\
FBS, mol/l & $6.21 \pm 2.84$ & $5.43 \pm 1.98$ & $2.106 \times 10^{-5} \mathrm{a}$ \\
\hline Characteristic & Cases (n=322) & Controls $(\mathrm{n}=473)$ & $\mathrm{p} \mathrm{value}$ \\
& $\mathrm{n}(\%) \mathrm{yes}$ & $\mathrm{n}(\%)$ yes & \\
\hline Males & $175(54.3)$ & $252(53.2)$ & $0.766^{\mathrm{b}}$ \\
DM & $112(34.8)$ & $79(16.7)$ & $1.497 \times 10^{-8} \mathrm{~b}$ \\
HBP & $185(57.5)$ & $77(16.3)$ & $7.858 \times 10^{-34} \mathrm{~b}$ \\
Smoking habit & $89(27.6)$ & $75(15.9)$ & $5.561 \times 10^{-5} \mathrm{~b}$ \\
Drinking status & $81(25.2)$ & $103(21.8)$ & $0.267^{\mathrm{b}}$ \\
\hline
\end{tabular}

aThe p value was calculated by the unpaired Student's t-test.

${ }^{\mathrm{b}}$ The $\mathrm{p}$ value was calculated by the chi square test.

\section{Results}

Baseline data of the cases and controls are shown in table 1. The levels of TC, BMI, and fasting blood sugar were significantly higher in cases than in controls (all p < 0.05). Notably, cases had a higher prevalence of diabetes mellitus, hypertension, and smoking habit (all $\mathrm{p}<$ 0.05). In our sample, rs8050136 and rs9939609 are in tight linkage disequilibrium: the D' value between the two SNPs is 0.99 , and the $r^{2}$ is 0.95 . Genotype frequency distribution and association with LAA stroke susceptibility for rs17782313 were summarized in table 2 . Genotype frequency distribution and association with LAA stroke susceptibility for rs8050136 and rs9939609 was summarized in supplementary table 2 and 3 (available at http://www. karger.com/ProdukteDB/produkte.asp?doi=448588), respectively. No differences were observed in genotype frequencies of polymorphisms of MC4R (rs17782313) or FTO (rs8050136 or rs9939609) between LAA stroke patients and control subjects (all p > 0.05).

In a stratified analysis according to the smoking status, no differences were observed in genotype frequencies of variants (rs8050136 or rs9939609) of FTO between LAA stroke patients and control subjects in the smoker or nonsmoker subgroups (all $\mathrm{p}>0.05$ ); Though rs17782313 of the $M C 4 R$ was not associated with LAA stroke in nonsmokers ( $p>0.05$ ), rs17782313 of the $M C 4 R$ was significantly associated with LAA stroke susceptibility in smokers ( $\mathrm{p}<0.05)$. The association between MC4R locus (rs17782313) and LAA stroke susceptibility in the smoker subgroup remained significant after adjustment for the covariates (status of diabetes mellitus, hypertension, and overweight). (rs17782313: $p=0.020$, OR $(95 \% \mathrm{CI})=1.55(1.07-2.23))$.

The results of the MDR analysis are summarized in table 3 . The best model was the model composed of MC4R locus (rs17782313), hypertension, and smoking habit, suggesting that the genetic and conventional risk factors together contribute to the etiology of LAA stroke. This model displayed the highest level of testing balance accuracy (0.7151) and a cross-validation consistency of 10/10. The results of MDR analysis revealed that the synergistic effects model of $M C 4 R$ locus (rs17782313), hypertension, and smoking habit may increase the risk of LAA stroke statistically, and the results of the permutation test were consistent (with 1,000 permu- 
DOI: $10.1159 / 000448588$ (c) 2016 The Author(s). Published by S. Karger GmbH, Freiburg www.karger.com/ofa

Song et al.: Evaluation of the Obesity Genes FTO and MC4R for Contribution to the Risk of Large Artery Atherosclerotic Stroke in a Chinese Population

Table 2. Genotype frequency distribution and association with LAA stroke susceptibility for the SNP (rs17782313)

\begin{tabular}{|c|c|c|c|c|}
\hline \multirow[t]{2}{*}{ Genotype } & \multicolumn{2}{|c|}{ Genotype frequencies, $\%$} & \multirow[t]{2}{*}{ OR $(95 \% \mathrm{CI})^{\mathrm{a}}$} & \multirow[t]{2}{*}{ p value ${ }^{a}$} \\
\hline & case, n (\%) & control, n (\%) & & \\
\hline \multicolumn{5}{|c|}{ Total participants } \\
\hline TT & $223(0.693)$ & $305(0.645)$ & $1.154(0.968-1.376)$ & 0.111 \\
\hline $\mathrm{TC}$ & $87(0.270)$ & $154(0.325)$ & & \\
\hline $\mathrm{CC}$ & $12(0.037)$ & $14(0.030)$ & & \\
\hline \multicolumn{5}{|c|}{ Non-smoker subgroup } \\
\hline $\mathrm{TT}$ & $160(68.7 \%)$ & $264(66.3 \%)$ & $1.060(0.866-1.297)$ & 0.571 \\
\hline $\mathrm{TC}$ & $64(27.5 \%)$ & $12(30.2 \%$ & & \\
\hline $\mathrm{CC}$ & $9(3.8 \%)$ & $14(3.5 \%)$ & & \\
\hline \multicolumn{5}{|c|}{ Smoker subgroup } \\
\hline TT & $63(70.8 \%)$ & $41(54.7 \%)$ & $1.55(1.07-2.23)$ & 0.020 \\
\hline $\mathrm{TC}$ & $23(25.8 \%)$ & $34(45.3 \%)$ & & \\
\hline $\mathrm{CC}$ & $3(3.4 \%)$ & $0(0 \%$ & & \\
\hline
\end{tabular}

LAA stroke = Large artery atherosclerotic stroke; CI =confidence interval; OR = odds ratio; SNPs = singlenucleotide polymorphisms (only associated SNPs are shown).

${ }^{\mathrm{a}} \mathrm{OR}(95 \% \mathrm{CI})$ and $\mathrm{p}$ value for the additive genetic model after adjustment for covariates (hypertension, overweight, diabetes).

Table 3. MDR models of loci and conventional risk factors for LAA stroke susceptibility

\begin{tabular}{llllll}
\hline Model & $\begin{array}{l}\text { Training } \\
\text { Bal.Acc }\end{array}$ & $\begin{array}{l}\text { Testing } \\
\text { Bal.Acc }\end{array}$ & $\begin{array}{l}\text { CV } \\
\text { consistency }\end{array}$ & p value & $\begin{array}{l}\text { p value for } \\
\text { permutation test }\end{array}$ \\
\hline Hypertension & 0.7059 & 0.7059 & $10 / 10$ & $<0.0001$ & 0.001 \\
Rs17782313 hypertension & 0.7059 & 0.7044 & $7 / 10$ & $<0.0001$ & 0.001 \\
Rs17782313 hypertension smoking habit & 0.7151 & 0.7151 & $10 / 10$ & $<0.0001$ & 0.001 \\
\hline
\end{tabular}

MDR = Multifactor dimensionality reduction; LAA stroke = large artery atherosclerotic stroke; Training Bal.Acc = training balanced accuracy; Testing Bal Acc $=$ testing balanced accuracy; $\mathrm{CV}$ consistency $=$ crossvalidation consistency.

tations $\mathrm{p}$ value is 0.001 ). In MDR analysis, the 3D combinations of $M C 4 R$ locus (rs17782313), hypertension, and smoking habit were classified into high- or low-risk groups. Background shading within each cell indicated the risk to LAA stroke of each given combination. High-risk groups are indicated by cells filled with dark shading, low-risk groups by cells filled with light shading (as shown in fig. 1). Based on the chi-square test, the OR of the high-risk combinations of the synergistic effects model indicated a 6.57-fold increased risk of LAA stroke $(\mathrm{p}<0.0001$, OR $(95 \% \mathrm{CI})=6.57$ (4.79-9.01)). Then, we used the software 'PS: Power and Sample Size Calculation (PS: version: 3.0.43)' to calculate the statistical power for our study. In assumption that OR is 1.582 , a threshold power value of $80 \%$ will be detected for our sample size. As the OR value is 6.57 in our MDR analysis, the statistical power according to our sample size is nearly 1.0. These results confirmed that MDR method is an efficient and powerful statistical tool for detecting synergistic interactions while avoiding the 'dimension curse', especially for detecting synergistic interactions in case-control studies using relatively small sample sizes. 
Song et al.: Evaluation of the Obesity Genes FTO and MC4R for Contribution to the Risk of Large Artery Atherosclerotic Stroke in a Chinese Population

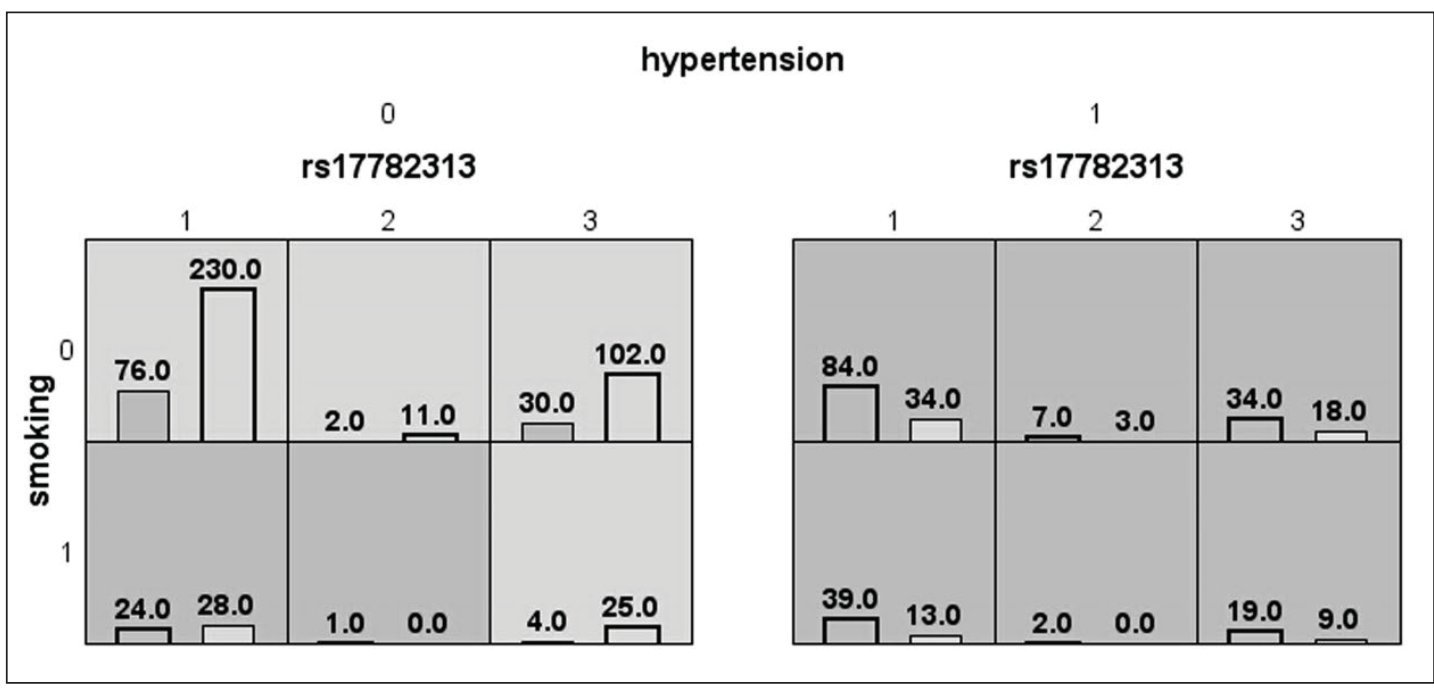

Fig. 1. The best model of factors contributing to LAA stroke identified by MDR. Notation: rs17782313 1/2/ $3=$ TT/CC/TC. Smoking 0/1 = non-smoker/smoker. Hypertension 0/1 = non-hypertension/hypertension. The best model which is composed of MC4R locus (rs17782313), hypertension, and smoking habit may increase the risk of LAA stroke statistically. The number in the left bar of each cell represents the number of LAA stroke patients with the given combination, which is indicated with bar filled with dark shading. While the number in the right bar of each cell represents the number of control subjects with the given combination, which is indicated with bar filled with light shading. Background shading within each cell indicated the risk to LAA stroke of each given combination. In MDR analysis, the 3D combinations of MC4R locus (rs17782313), hypertension, and smoking habit were classified into high- or low-risk groups. High-risk cells are indicated by dark shading, low-risk cells by light shading. Based on the chi-square test, the OR value of the high-risk combinations of the synergistic effects model indicated a 6.57-fold increased risk of LAA stroke $(\mathrm{p}<0.0001$, OR $(95 \% \mathrm{CI})=6.57(4.79-9.01))$.

\section{Discussion}

Identification of the Contribution of Synergistic Effect between Obesity Gene and Conventional Risk Factors to LAA Stroke Risk in a Chinese Population

China is a country with a high incidence of stroke, and intracranial atherosclerosis is more prevalent in the Chinese Han population than in Western populations [20-22]. Meanwhile, obesity is a well-established risk factor for LAA stroke, and many obesity-prone genes have been found in GWASs. Intriguingly, the associations between obesity-prone genes and LAA stroke in the Chinese Han population have not been studied previously. It was of interest to investigate the relationship between obesity-prone genes (such as FTO and MC4R) and LAA stroke in the Chinese Han population.

Our study revealed no evidence for association between single variants of FTO or MC4R and LAA stroke, which is in accordance with the report of the Mannheim-Heidelberg stroke study in Germany [15]. However, our stratified analysis also showed evidence that rs 17782313 of $M C 4 R$ was associated with LAA stroke susceptibility in a smoker subgroup based on a stratified analysis according to the smoking status. The association between $M C 4 R$ locus (rs17782313) and LAA stroke susceptibility in the smoker subgroup remained significant after adjusting for covariates. Thus, the result of stratified analysis indicated a possible synergistic effect between $M C 4 R$ polymorphism and smoking habit. To further identify higherorder genetic-conventional risk factor interactions in our samples, we applied the MDR 
Song et al.: Evaluation of the Obesity Genes FTO and MC4R for Contribution to the Risk of Large Artery Atherosclerotic Stroke in a Chinese Population

method, which is regarded as an advanced and promising strategy to assess gene-environment and gene-gene interactions in the etiology analysis of complex diseases [18, 23], such as cardiovascular disease and cerebrovascular disease. Interestingly, our MDR analysis revealed that the combination of $M C 4 R$ locus (rs17782313), hypertension, and smoking habit significantly increased the risk of LAA stroke $(\mathrm{p}<0.0001$, OR $(95 \% \mathrm{CI})=6.57(4.79-9.01))$. The results of MDR analysis indicated a prominent synergistic interplay of gene and conventional risk factors with regard to LAA stroke risk.

The Finding of Synergistic Effects between MC4R and Conventional Risk Factors on Stroke Risk Provide New Insight into the Genetic Architecture of LAA Stroke

$M C 4 R$ is located on chromosome 18q22. The protein encoded by this gene is a G-protein coupled receptor that is expressed in the central nervous system and has a crucial role in regulating the balance between feeding behavior and energy expenditure. The alteration of MC4R function or expression may result in imbalance of the energy homeostasis, body fat accumulation, and eventually obesity. Loos RJ et al. [11] analyzed genome-wide association data from 16,876 individuals of European descent; the study revealed the strongest association signal ( $\mathrm{rs} 17782313, \mathrm{p}=2.9 \times 10^{-6}$ ) mapped $188 \mathrm{~kb}$ downstream of $M C 4 R$ coding sequence, which established that common variants near MC4R influence fat mass, weight, and obesity risk at the population level. The SNP location and patterns of phenotypic associations are consistent with effects mediated through altered MC4R function, although there is no research to prove that this SNP has a functional effect on MC4R at present. Further studies should be conducted to identify the causal variant and the underlying mechanisms of the identified association in the future. Furthermore, the result of a recent study focusing on the Chinese Han population indicated that rs17782313 of MC4R was associated with childhood obesity [14], which is consistent with the results of GWASs of European descent. Additionally, previous studies revealed evidence that rs17782313 affects not only the levels of plasma lipids including TC and LDL-C [24], but also glycemic traits and susceptibility of type 2 diabetes in the Chinese Han population [25]. A recent study also revealed that rs17782313 variants of $M C 4 R$ may be associated with nocturnal blood pressure levels in the Chinese Han population, indicating a possible role of $M C 4 R$ on blood pressure regulation [26].

The remarkable influences of $M C 4 R$ on obesity and related metabolic syndromes (ranging from disturbance of blood glucose and lipids to elevated blood pressure levels) may increase the susceptibility of atherosclerosis, the underlying pathologic change of LAA stroke. A previous report [15] has analyzed the contribution of $M C 4 R$ to stroke, but the association between $M C 4 R$ polymorphisms and stroke has not been convincing. Moreover, the result of our single-locus findings concerning the association between $M C 4 R$ gene polymorphisms and LAA stroke was also negative. Although we did not find any significant main effect, we did report an association between LAA stroke and the SNP (rs17782313) in smoker subgroups in the stratified analysis according to smoking status. It is the first study indicating that rs17782313 may contribute to stroke risk. Furthermore, we attribute the difference between our results and previous studies to either racial difference or a synergistic effect between rs17782313 and smoking on LAA stroke risk. It is well known that many genetic and environmental risk factors may take part in the disease pathogenesis of complex diseases such as LAA stroke. And it is believed that the more risk factors are present, the higher is the susceptibility to come down with this complex disease. Thus, we postulated that the MC4R gene is a lowpenetrance susceptibility marker that exerts a minor marginal effect on LAA stroke, and this gene may impact stroke risk via a synergistic mechanism. Our MDR analysis confirmed that the three-way combination of MC4R polymorphism (rs17782313), hypertension, and smoking habit contributes significantly to the risk of LAA stroke in the Chinese Han population, which is consistent with previous reports demonstrating the synergistic effect of gene and conven- 
Song et al.: Evaluation of the Obesity Genes FTO and MC4R for Contribution to the

tional risk factors by increasing the risk to suffer from complex disease $[27,28]$. The revealing finding of MDR analysis will provide new insights into the genetic architecture of LAA stroke.

The Obesity-Prone Gene MC4R Contributes to the Risk of LAA Stroke via a Synergistic

Mechanism, Which May Provide Enlightenment for the Prevention of LAA Stroke

From the perspective of stroke prevention, the risk factors can be divided into various categories: congenital or acquired, modifiable or not, classical or non-classical $[29,30]$. It is well known that interventions of acquired and modifiable risk factors, such as managements of hypertension and smoking cessation, are efficient strategies for stroke prevention [31-33]. Our finding revealed that obesity-prone genes such as $M C 4 R$ contribute to the risk of LAA stroke via a synergistic mechanism, indicating that congenital risk factors, such as MC4R polymorphism, may also play an important role in the pathogenesis of LAA stroke. Although the $M C 4 R$ polymorphism is not modifiable for clinic practice at present, several treatment options have been investigated in subjects with $M C 4 R$ mutation-related obesities. A study showed that an intensive lifestyle intervention induces similar weight reduction in $M C 4 R$ mutation carriers in comparison to $M C 4 R$ mutation non-carriers [34]. However, long-term body weight maintenance is hardly ever achieved in $M C 4 R$ mutation carriers [35]. Meanwhile, a variety of in vitro experimental studies on the management of $M C 4 R$ disruption have been conducted. In vitro studies with melanocortin agonists showed that mutated human $M C 4 R$ with impaired endogenous agonist functional response can be activated by some of these agonists and may represent a valuable therapeutic target [36]. Furthermore, another study indicated that pharmacological chaperones that recover cell surface expression of MC4R may represent a candidate for the development of a targeted therapy suitable for patients with MC4R-deficient obesity [37]. It is postulated that chemical chaperones and pharmacological agonists efficiently restore cell surface expression and that endogenous agonist response of mutated $M C 4 R$ may be effective in the treatment of adiposity. However, further research in the development of drugs for $M C 4 R$ variations is needed. Although pharmacotherapy targeting obesityprone genes such as $M C 4 R$ has not clinically been tested in the prevention of cerebrovascular disease, identification of pertinent genes may unravel new therapeutic strategies to counter the influence of gene polymorphism on obesity and hence minimize its notable vascular complications, such as LAA stroke.

\section{Conclusion}

In conclusion, our results revealed no evidence of association between FTO polymorphism and LAA stroke. However, our study indicated that a synergistic effect of MC4R polymorphism (rs17782313), hypertension, and smoking habit contribute significantly to the risk of LAA stroke in the Chinese Han population. The finding revealed that the obesity-prone gene $M C 4 R$ contributes to the risk of LAA stroke via a synergistic mechanism, which will provide new insights into the genetic architecture of LAA stroke. We believe these findings will eventually guide a promising prevention strategy of LAA stroke in the future.

\section{Funding}

This study was supported by The New Xiangya Talent Project of the Third Xiangya Hospital of Central South University of China (JY201608). 


\begin{tabular}{l} 
Obes Facts 2016;9:353-362 \\
\hline DOI: $10.1159 / 000448588$ \\
$\begin{array}{l}\text { (c) } 2016 \text { The Author(s). Published by S. Karger GmbH, Freiburg } \\
\text { www.karger.com/ofa }\end{array}$ \\
\hline
\end{tabular}

Song et al.: Evaluation of the Obesity Genes FTO and MC4R for Contribution to the Risk of Large Artery Atherosclerotic Stroke in a Chinese Population

\section{Disclosure Statement}

The author(s) declared no potential conflicts of interest with respect to the research, authorship, and/ or publication of this article.

\section{References}

1 Flossmann E, Rothwell PM: Family history of stroke in patients with transient ischemic attack in relation to hypertension and other intermediate phenotypes. Stroke 2005;36:830-835.

2 Meschia JF: Ischemic stroke as a complex genetic disorder. Semin Neurol 2006;26:49-56.

-3 Yatsuya H, Toyoshima H, Yamagishi K, Tamakoshi K, Taguri M, Harada A, Ohashi Y, Kita Y, Naito Y, Yamada M, Tanabe N, Iso H, Ueshima H; Japan Arteriosclerosis Longitudinal Study (JALS) Group: Body mass index and risk of stroke and myocardial infarction in a relatively lean population: meta-analysis of 16 Japanese cohorts using individual data. Circ Cardiovasc Qual Outcomes 2010;3:498-505.

4 Bodenant M, Kuulasmaa K, Wagner A, Kee F, Palmieri L, Ferrario MM, Montaye M, Amouyel P, Dallongeville J: Measures of abdominal adiposity and the risk of stroke: the MOnica Risk, Genetics, Archiving and Monograph (MORGAM) study. Stroke 2011;42:2872-2877.

5 Karcher HS, Holzwarth R, Mueller HP, Ludolph AC, Huber R, Kassubek J, Pinkhardt EH: Body fat distribution as a risk factor for cerebrovascular disease: an MRI-based body fat quantification study. Cerebrovasc Dis 2013; 35:341-348.

6 Lucke-Wold BP, Turner RC, Lucke-Wold AN, Rosen CL, Huber JD: Age and the metabolic syndrome as risk factors for ischemic stroke: improving preclinical models of ischemic stroke. Yale J Biol Med 2012;85:523539.

7 Flossmann E, Schulz UG, Rothwell PM: Potential confounding by intermediate phenotypes in studies of the genetics of ischaemic stroke. Cerebrovasc Dis 2005;19:1-10.

8 Paquot N, Tappy L: Adipocytokines: link between obesity, type 2 diabetes and atherosclerosis. Rev Med Liege 2005;60:369-373.

-9 Fesinmeyer MD, North KE, Ritchie MD, Lim U, Franceschini N, Wilkens LR, Gross MD, Bůžková P, Glenn K, Quibrera PM, Fernández-Rhodes L, Li Q, Fowke JH, Li R, Carlson CS, Prentice RL, Kuller LH, Manson JE, Matise TC, Cole SA, Chen CT, Howard BV, Kolonel LN, Henderson BE, Monroe KR, Crawford DC, Hindorff LA, Buyske S, Haiman CA, Le Marchand L, Peters U: Genetic risk factors for BMI and obesity in an ethnically diverse population: results from the population architecture using genomics and epidemiology (PAGE) study. Obesity (Silver Spring) 2013;21:835-846.

-10 Frayling TM, Timpson NJ, Weedon MN, Zeggini E, Freathy RM, Lindgren CM, Perry JR, Elliott KS, Lango H, Rayner NW, Shields B, Harries LW, Barrett JC, Ellard S, Groves CJ, Knight B, Patch AM, Ness AR, Ebrahim S, Lawlor DA, Ring SM, Ben-Shlomo Y, Jarvelin MR, Sovio U, Bennett AJ, Melzer D, Ferrucci L, Loos RJ, Barroso I, Wareham NJ, Karpe F, Owen KR, Cardon LR, Walker M, Hitman GA, Palmer CN, Doney AS, Morris AD, Smith GD, Hattersley AT, McCarthy MI: A common variant in the FTO gene is associated with body mass index and predisposes to childhood and adult obesity. Science 2007;316:889-894.

11 Loos RJ, Lindgren CM, Li S, et al: Common variants near MC4R are associated with fat mass, weight and risk of obesity. Nat Genet 2008;40:768-775.

12 Monda KL, Chen GK, Taylor KC, et al: A meta-analysis identifies new loci associated with body mass index in individuals of African ancestry. Nat Genet 2013;45:690-696.

13 Xu Y, Ling J, Yang M, Wang H, Zhang S, Zhang X, Zhu Y: Rs7206790 and rs11644943 in FTO gene are associated with risk of obesity in Chinese school-age population. PLoS One 2014;9:e108050.

14 Lv D, Zhang DD, Wang H, Zhang Y, Liang L, Fu JF, Xiong F, Liu GL, Gong CX, Luo FH, Chen SK, Li ZL, Zhu YM: Genetic variations in SEC16B, MC4R, MAP2K5 and KCTD15 were associated with childhood obesity and interacted with dietary behaviors in Chinese school-age population. Gene 2015;560:149-155.

-15 Winter Y, Back T, Scherag A, Linseisen J, Rohrmann S, Lanczik O, Hinney A, Scherag S, Neumaier M, Ringleb PA, Dodel R, Hebebrand J: Evaluation of the obesity genes FTO and MC4R and the type 2 diabetes mellitus gene TCF7L2 for contribution to stroke risk: the Mannheim-Heidelberg Stroke Study. Obes Facts 2011;4:290-296.

16 Adams HP Jr, Bendixen BH, Kappelle LJ, Biller J, Love BB, Gordon DL, Marsh EE 3rd: Classification of subtype of acute ischemic stroke. Definitions for use in a multicenter clinical trial.TOAST. Trial of Org 10172 in Acute Stroke Treatment. Stroke 1993;24:35-41.

$\checkmark 17$ Meyer K, Ueland PM: Use of matrix-assisted laser desorption/ionization time-of-flight mass spectrometry for multiplex genotyping. Adv Clin Chem 2011;53:1-29.

18 Hahn LW, Ritchie MD, Moore JH: Multifactor dimensionality reduction software for detecting gene-gene and gene-environment interactions. Bioinformatics 2003;19:376-382.

$\checkmark 19$ Kwon MS, Kim K, Lee S, Park T: cuGWAM: genome-wide association multifactor dimensionality reduction using CUDA-enabled high-performance graphics processing unit. Int J Data Min Bioinform 2012;6:471-481.

20 Jiang B, Wang WZ, Chen H, Hong Z, Yang QD, Wu SP, Du XL, Bao QJ: Incidence and trends of stroke and its subtypes in China: results from three large cities. Stroke 2006;37:63-68. 
Song et al.: Evaluation of the Obesity Genes FTO and MC4R for Contribution to the Risk of Large Artery Atherosclerotic Stroke in a Chinese Population mutations in obese Czech children: studies of prevalence, phenotype development, weight reduction response, and functional analysis. J Clin Endocrinol Metab 2007;92:3689-3696.

-35 Reinehr T, Hebebrand J, Friedel S, Toschke AM, Brumm H, Biebermann H, Hinney A: Lifestyle intervention in obese children with variations in the melanocortin 4 receptor gene. Obesity (Silver Spring) 2009;17:382-389.

36 Roubert P, Dubern B, Plas P, Lubrano-Berthelier C, Alihi R, Auger F, Deoliveira DB, Dong JZ, Basdevant A, Thurieau C, Clément K: Novel pharmacological MC4R agonists can efficiently activate mutated MC4R from obese patient with impaired endogenous agonist response. J Endocrinol 2010;207:177-183.

-37 Rene P, Le Gouill C, Pogozheva ID, Lee G, Mosberg HI, Farooqi IS, Valenzano KJ, Bouvier M: Pharmacological chaperones restore function to MC4R mutants responsible for severe early-onset obesity. J Pharmacol Exp Ther 2010;335:520-532. 